\title{
THE DISTORTION THEOREM FOR QUASICONFORMAL MAPPINGS, SCHOTTKY'S THEOREM AND HOLOMORPHIC MOTIONS
}

\author{
G. J. MARTIN
}

(Communicated by Albert Baernstein II)

\begin{abstract}
We prove the equivalence of Schottky's theorem and the distortion theorem for planar quasiconformal mappings via the theory of holomorphic motions. The ideas lead to new methods in the study of distortion theorems for quasiconformal mappings and a new proof of Teichmüller's distortion theorem.
\end{abstract}

\section{INTRODUCTION}

In a previous joint paper with F.W. Gehring [9] a connection between two classical theorems of complex analysis was pointed out; namely Schottky's Theorem and the Distortion Theorem for planar quasiconformal mappings. In that paper we were studying the motions of Julia sets of a certain quadratic polynomial in order to prove an inequality concerning two-generator discrete groups of Möbius transformations. We found that the best possible estimates could be obtained either by using the sharp form of Schottky's Theorem or the sharp form of the Distortion Theorem for quasiconformal mappings. It is the purpose of this paper to show how the equivalence of these two well known results arises as a consequence of the theory of holomorphic motions. As an application we will prove a sharp extension of a classical distortion estimate for quasiconformal mappings. We then show how the methods can be used to give a new proof of Teichmüller's classical result on the distortion of quasiconformal mappings, a result from which many other distortion theorems are derived. We are indebted to A. Hinkkanen for pointing this connection out to us. It is also interesting to look at S. Agard's early paper on distortion theorems for quasiconformal mappings [2]. Here one sees, with hindsight, a connection between distortion theorems and Schottky's theorem and perhaps the germ of an idea relating to holomorphic motions. However at that time very little of the modern theory was available.

The reader will see that there are many other applications of this circle of ideas, and that other sharp estimates for quasiconformal mappings can be obtained using fundamentally new methods. Roughly, it turns out that the distortion estimates of a suitably normalised quasiconformal mapping are controlled by the growth of an analytic mapping (usually a universal covering map). Two instances are Mori's

Received by the editors March 20, 1995 and, in revised form, September 28, 1995.

1991 Mathematics Subject Classification. Primary 30C60.

This research was supported in part by grants from the New Zealand Foundation for Research, Science and Technology and the Australian Research Council.

(C)1997 American Mathematical Society 
distortion theorem, where the distortion is controlled by the growth of the universal covering map $\Delta \rightarrow \mathbb{C} \backslash\{0,1\}$ and the quasiconformal Schwarz Lemma, where the distortion is controlled by the universal covering map $\Delta \rightarrow \Delta \backslash\{0\}$. We shall consider distortion estimates for "overnormalised" quasiconformal mappings using the equivariant version of the $\lambda$-lemma [8] in a sequel.

In other directions K. Astala, S. Rhode and O. Schramm have used these and related ideas to estimate the rate of change of Hausdorff dimension under holomorphic motions. They obtain good new estimates on the Hausdorff dimension of quasicircles. Conversely, M. Vuorinen has used new distortion estimates for quasiconformal mappings to provide improved estimates in Schottky's theorem.

We begin with a few basic concepts. A fuller account of the theory of quasiconformal mappings can be found in Ahlfors' book [1] (see also Lehto-Virtanen [13]), and some of the basic facts about quasiconformal mappings and Teichmüller spaces we shall use can be found in Lehto's book [12].

Definition 1.1. A sense preserving homeomorphism $f$ of a domain $\Omega \subset \mathbb{C}$ is $K$ quasiconformal (abbreviated $K$-qc), $1 \leq K<\infty$, if $f$ is an $L^{2}$-solution of the equation

$$
\bar{\partial} f=\mu \partial f
$$

where $\mu$ is a Borel measurable function with

$$
\|\mu\|_{\infty} \leq \frac{K-1}{K+1}<1 .
$$

The equation (1) is known as the Beltrami equation and has a long history going back to Gauss, who studied it with smooth $\mu$ in investigating the problem of existence of isothermal coordinates on a surface. The Beltrami equation generalises the Cauchy-Riemann equations, obtained when $\mu=0$. The coefficient function $\mu$ is called the complex dilatation of $f$. The condition that the solution is in $L^{2}$ can be relaxed to the assumption that $f$ is differentiable a.e. $\Omega$ and is an $L^{1}$ solution. It is a deep result known as "the existence theorem for quasiconformal mappings" (or more recently as "the measurable Riemann mapping theorem") that every measurable $\mu$ defined in a domain $\Omega$ with $\|\mu\|_{\infty}<1$ can be realised as the complex dilatation of a quasiconformal mapping (a.e. $\Omega$ ). From the definition it follows that the following infinitesimal distortion estimate holds:

$$
\limsup _{r \rightarrow 0} \frac{\max _{|h|=r}|f(z+h)-f(z)|}{\min _{|h|=r}|f(z+h)-f(z)|}=H(z) \leq K \quad \text { a.e. } \Omega .
$$

The distortion theory of quasiconformal mappings is largely concerned with the problem of obtaining global distortion bounds on quasiconformal mappings from the local bounds of (3). The best-known of these is Mori's theorem which asserts that for a $K$-quasiconformal mapping $f: \mathbb{C} \rightarrow \mathbb{C}$ there is a $\mathcal{K}$ depending only on $K$ such that

$$
\max _{|h|=r}|f(z+h)-f(z)| \leq \mathcal{K} \min _{|h|=r}|f(z+h)-f(z)| .
$$

Thus bounds on the number $\mathcal{K}$ give control on the distortion of an arbitrary circle of radius $r$ centered at any point $z \in \mathbb{C}$ under a $K$-quasiconformal mapping. Clearly this estimate is invariant under translations and dilations of the homeomorphism 
$f$. Thus we normalise quasiconformal mappings via these transformations and we define for $t \in[0,1)$ the family of normalised quasiconformal mappings

$$
\mathcal{Q} C(t)=\left\{f: \mathbb{C} \rightarrow \mathbb{C} \mid f \text { is } K=\frac{1+t}{1-t}-\mathrm{qc}, f(0)=0, f(1)=1\right\} .
$$

Schottky's theorem is concerned with the rate of growth of analytic maps of the unit disk $\Delta=\{z \in \mathbb{C}:|z|<1\}$ into the complex plane omitting two finite values, which we assume to be 0 and 1 . Thus we define

$$
\mathcal{A}(r)=\{\varphi: \Delta \rightarrow \mathbb{C} \backslash\{0,1\}: \varphi \text { analytic, }|\varphi(0)|=r\} .
$$

We then define the two extremal functions

$$
\Psi_{\mathcal{Q} C}(t, r)=\sup \{|f(z)|: f \in \mathcal{Q} C(t),|z|=r\}
$$

and

$$
\Psi_{\mathcal{A}}(t, r)=\sup \{|\varphi(z)|: \varphi \in \mathcal{A}(r),|z|=t\} .
$$

An explicit formula for $\Psi_{\mathcal{Q} C}(t, r)$ in terms of the Grötzsch function can be found in [2]. The duality between Schottky's theorem and the distortion theorem is more appropriately explained in the proof of the following theorem than in its precise statement.

Theorem 1.1. For all $t \in[0,1)$ and $r>0$

$$
\Psi_{\mathcal{Q} C}(t, r)=\Psi_{\mathcal{A}}(t, r) .
$$

In order to prove this result we shall need the concept of holomorphic motions and the "extended $\lambda$-lemma". Basically a holomorphic motion is a holomorphically parametrised isotopy of a subset of the Riemann sphere $\overline{\mathbb{C}}=\mathbb{C} \cup\{\infty\}$. Here is the formal definition.

Definition 1.2. A holomorphic motion of a set $A \subset \overline{\mathbb{C}}$ is a map $h: \Delta \times A \rightarrow \overline{\mathbb{C}}$ such that

(i) for each fixed $a \in A$, the map $\lambda \rightarrow h(\lambda, a)$ is holomorphic in $\Delta$,

(ii) for each fixed $\lambda \in \Delta$, the map $z \rightarrow h(\lambda, a)=h_{\lambda}(a)$ is an injection,

(iii) the mapping $h_{0}$ is the identity on $A$.

Note that there is no assumption regarding the continuity of $h$ as a function of $a$ or the pair $(\lambda, a)$. That such continuity occurs is a consequence of the following remarkable $\lambda$-lemma of Mañé-Sad-Sullivan [15]. We give here the result as extended by Slodkowski [16]; see also [4] and [7].

Theorem 1.2. If $h: \Delta \times A \rightarrow \overline{\mathbb{C}}$ is a holomorphic motion of $A \subset \mathbb{C}$, then $h$ has an extension to $H: \Delta \times \overline{\mathbb{C}} \rightarrow \overline{\mathbb{C}}$ such that

(i) $H$ is a holomorphic motion of $\overline{\mathbb{C}}$,

(ii) $H$ is continuous in $\Delta \times \overline{\mathbb{C}} \rightarrow \overline{\mathbb{C}}$,

(iii) $H_{\lambda}: \overline{\mathbb{C}} \rightarrow \overline{\mathbb{C}}$ is a $K$-quasiconformal homeomorphism of $\mathbb{C}$ with $K \leq \frac{1+|\lambda|}{1-|\lambda|}$ for each $\lambda \in \Delta$.

Holomorphic motions arise naturally in the study of complex dynamical systems, for example the iteration of polynomials or rational functions in the complex plane, and in the study of Kleinian groups. This is because as one holomorphically varies the parameters of the dynamical system, for example the coefficients of the polynomials or the coefficients of the generating Möbius transformations, periodic cycles and their eigenvalues also vary analytically in the former case and loxodromic fixed 
points vary analytically in the latter case. Using the dynamics one can place restrictions on the parameters of the system so that this analytic variation is actually a holomorphic motion, and then density results imply holomorphic variation of the Julia set of the rational mapping or the limit set of a Kleinian group as the case may be.

\section{Proof of Theorem 1.1}

We prove the theorem in two parts. First we show $\Psi_{\mathcal{Q} C}(t, r) \geq \Psi_{\mathcal{A}}(t, r)$. Thus let $\varphi: \Delta \rightarrow \mathbb{C} \backslash\{0,1\}$ be an analytic function with $|\varphi(0)|=r$. Evidently the function

$$
h(\lambda, a)= \begin{cases}\varphi(\lambda) & \text { if } a=\varphi(0), \\ 0 & \text { if } a=0, \\ 1 & \text { if } a=1, \\ \infty & \text { if } a=\infty,\end{cases}
$$

is a holomorphic motion of the four-point set $\{0,1, \infty, \varphi(0)\}$. Extend this to a holomorphic motion $H$ of $\overline{\mathbb{C}}$ and consider the circle $|z|=r$. For each $\lambda \in \Delta$ the mapping $H_{\lambda}(\cdot)$ is a $K=\frac{1+|\lambda|}{1-|\lambda|}$-quasiconformal mapping of $\mathbb{C}$, and $H_{\lambda}(\varphi(0))$ lies on the image of the circle $|z|=r$. Thus, by definition,

$$
|\varphi(\lambda)|=\left|H_{\lambda}(\varphi(0))\right| \leq \Psi_{\mathcal{Q} C}(t, r)
$$

since

$$
t=\frac{K-1}{K+1}=|\lambda| \text {. }
$$

As $\varphi$ is arbitrary, we have shown $\Psi_{\mathcal{Q} C}(t, r) \geq \Psi_{\mathcal{A}}(t, r)$.

We now show $\Psi_{\mathcal{Q} C}(t, r) \leq \Psi_{\mathcal{A}}(t, r)$. This requires a little more theory. Let $f(z)$ be a $K$-quasiconformal homeomorphism of $\mathbb{C}$ with complex dilatation $\mu_{f}$,

$$
\left\|\mu_{f}\right\|_{\infty}=\frac{K-1}{K+1}=t .
$$

For $|\lambda|<1$ let

$$
\mu_{\lambda}=\frac{\lambda}{t} \mu_{f}
$$

Thus $\mu_{t}=\mu_{f}$. Now let $f_{\lambda}(z)$ be the normalised quasiconformal mapping solving the Beltrami equation

$$
\bar{\partial} f_{\lambda}=\mu_{\lambda} \partial f_{\lambda}
$$

That is, the complex dilatation of $f_{\lambda}$ is $\mu_{\lambda}$. By the Ahlfors-Bers theory ([12] Chapter 5 ), the quasiconformal mappings $f_{\lambda}$ depend holomorphically on the variable $\lambda$. In particular, for each $z \in \mathbb{C}$ the mappings $\varphi_{z}: \lambda \rightarrow f_{\lambda}(z)$ are analytic. Notice too that $f_{t}(z)=f(z)$, and that $f_{0}(z)=z$ by the uniqueness part of the existence theorem for quasiconformal mappings. Since $f(0)=0, f(1)=1$ and $f(\infty)=\infty$, this analytic mapping has values in $\mathbb{C} \backslash\{0,1\}$. Thus for any $z \in \mathbb{C}$ with $|z|=r$ we have, by definition,

$$
|f(z)|=\left|f_{t}(z)\right|=\left|\varphi_{z}(t)\right| \leq \Psi_{\mathcal{A}}\left(t,\left|\varphi_{z}(0)\right|\right)=\Psi_{\mathcal{A}}(t, r)
$$

since $\left|\varphi_{z}(0)\right|=\left|f_{0}(z)\right|=|z|=r$. 


\section{An Application}

Because of Theorem 1 , for $0 \leq t<1$ and $r>0$ we may define

$$
\Psi(t, r)=\Psi_{\mathcal{A}}(t, r)=\Psi_{\mathcal{Q} C}(t, r) .
$$

In his paper [10] J. Hempel gave the following implicit formula for the function $\Psi(t, r)$ :

$$
\int_{r}^{\Psi} \rho(-x) d x=\log \frac{1+t}{1-t}
$$

where $\rho(\cdot)$ is the Poincaré density of the thrice punctured sphere $\mathbb{C} \backslash\{0,1\}$. The left hand side is of course just the hyperbolic distance between $-r$ and $-\Psi$ in $\mathbb{C} \backslash\{0,1\}$, and $\rho(\cdot)$ is related to the elliptic modular function $\lambda(\omega)$ via the formula

$$
\rho(\lambda(\omega))\left|\lambda^{\prime}(\omega)\right|=\frac{1}{\Im(\omega)} .
$$

It is from this that one is able to obtain estimates. Hempel gives, among others, the asymptotically sharp bounds,

- $\Psi(t, r)<\frac{1}{16}\left(r e^{\pi}\right)^{(1+t) /(1-t)}$ for $r \geq 1$,

- $\Psi(t, r)<\frac{1}{16}\left(e^{\pi}\right)^{(1+t) /(1-t)}$ for $r \leq 1$.

Presumably estimates like this were known to Teichmüller. More refined estimates occur in [3], where it is shown that for $K=(1+t) /(1-t)$

$$
\Psi(t, 1) \min \left\{r^{K}, r^{1 / K}\right\} \leq \Psi(t, r) \leq \Psi(t, 1) \max \left\{r^{K}, r^{1 / K}\right\} .
$$

Here when $r=1$ we have the usual distortion function for quasiconformal mappings with the better bound [13]

$$
\Psi(t, 1)=\frac{1}{16} e^{\pi(1+t) /(1-t)}-\frac{1}{2}+o(1)
$$

and $0<o(1) \leq 2 e^{-\pi(1+t) /(1-t)}$ by [14]. We also note that the sharp asymptotics for the distortion theorem for $K$ near 1 can be deduced from estimates on $\Psi(t, r)$ for $t$ near 0 . Easy estimates (using equation (11)) give

$$
\Psi(t, r) \approx r+\frac{2 t-4 t^{2}}{\rho(-r)}+O\left(t^{3}\right)
$$

for small $t$. Beurling and Ahlfors give the estimate $\Psi(t, 1) \leq e^{a t /(1-t)}$ with $a=$ $\left.2 \frac{d}{d t} \psi(t, 1)\right|_{t=0} \approx 8.76 \ldots[5]$. Because the sharp bounds for the analytic functions occur for negative real values, the proof of Theorem 1.1 shows that

$$
\Psi(t, r)=\max \{|f(-r)|: f \in \mathcal{Q} C(t)\},
$$

a result which is relatively clear by symmetry, but not so easy to prove directly; see [17]. We now state a sharp generalization of Mori's Theorem (the case $r=1$ ).

Theorem 3.1. Let $f: \mathbb{C} \rightarrow \mathbb{C}$ be a $K$-quasiconformal mapping. Then

$$
\max _{\theta \in[0,2 \pi]}\left|f\left(z_{0}+r e^{\imath \theta}\right)-f\left(z_{0}\right)\right| \leq \frac{1}{16} e^{\pi K} r^{K} \min _{\theta \in[0,2 \pi]}\left|f\left(z_{0}+e^{\imath \theta}\right)-f\left(z_{0}\right)\right|
$$

for all $z_{0} \in \mathbb{C}$ and $r>1$. 
Proof. Choose $\theta \in[0,2 \pi]$ so that

$$
\left|f\left(z_{0}+e^{\imath \theta}\right)-f\left(z_{0}\right)\right|=\min _{\theta \in[0,2 \pi]}\left|f\left(z_{0}+e^{\imath \theta}\right)-f\left(z_{0}\right)\right| .
$$

Then set

$$
g(z)=\frac{f\left(z_{0}+z e^{\imath \theta}\right)-f\left(z_{0}\right)}{f\left(z_{0}+e^{\imath \theta}\right)-f\left(z_{0}\right)}
$$

Then $g(0)=0, g(1)=1$ and $g$ is $K$-quasiconformal. Thus

$$
|g(z)| \leq \Psi\left(\frac{K-1}{K+1},|z|\right),
$$

from which the result follows from the estimates above.

Theorem 3.1 can also be deduced from [3]. There is of course a related result for $r<1$. Another known distortion result is easily proved using holomorphic motions as well. We sketch the proof of the following theorem.

Theorem 3.2. Let $f: \mathbb{C} \rightarrow \mathbb{C}$ be a $K$-quasiconformal mapping with $f(0)=0$, and let $z, w \in \mathbb{C}$ with $0<|w| \leq|z|$. Then

$$
\left|\frac{f(z)}{f(w)}\right| \leq \frac{e^{\pi K}}{16}\left|\frac{z}{w}\right|^{K}
$$

Proof. Drag $f$ back to the scalar multiplication $\zeta \rightarrow \alpha \zeta, \alpha=f(z) / z$, via a holomorphic motion as in the proof of Theorem 1.1 with the normalisations $f_{\lambda}(0)=$ $0, f_{\lambda}(z)=f(z)$. The traces of $z$ and $w$ give nonzero analytic mappings of the disk $\varphi_{z}$ and $\varphi_{w}$ such that $\varphi_{z}(\lambda) \neq \varphi_{w}(\lambda), \varphi_{z}(0)=\alpha z, \varphi_{w}(0)=\alpha w, \varphi_{z}(k)=f(z)$ and $\varphi_{w}(k)=f(w)$, where $k$ is the sup norm of the complex dilatation of $f$. Now the analytic mapping $\varphi_{z}(\lambda) / \varphi_{w}(\lambda)$ is valued in $\mathbb{C} \backslash\{0,1\}$, and Hempel's bound given above yields the desired result.

\section{TeichmülleR's Theorem}

There is a classical result of Teichmüller concerning the distortion of normalised quasiconformal mappings. We will show how our Theorem 1.1 is equivalent to this theorem. First, here is the statement of Teichmüller's Theorem.

Theorem 4.1. Let $\rho(z, w)$ denote the hyperbolic metric of constant curvature -4 in the thrice punctured sphere $\mathbb{C} \backslash\{0,1\}$. Let $f$ be a $K$-quasiconformal mapping of the Riemann sphere fixing 0,1 and $\infty$. Then for any $z \in \mathbb{C} \backslash\{0,1\}$ we have

$$
\rho(z, f(z)) \leq \log K
$$

Furthermore if $z, w \in \mathbb{C} \backslash\{0,1\}$ satisfy $\rho(z, w) \leq \log K$, then there is a $K$-quasiconformal map of the Riemann sphere fixing 0,1 and $\infty$ such that $w=f(z)$.

We prove the equivalence of Theorem 1.1 and Theorem 4.1 (more precisely, the proof of Theorem 1.1). 
Theorem 1.1 implies Theorem 4.1. Drag $f$ back to the identity via a normalised holomorphic motion as in the proof of Theorem 1.1. Let $\varphi$ denote the analytic disk which is the trace of the point $z$, so that $\varphi(0)=z$ and $\varphi(k)=f(z)$, where $k=(K-1) /(K+1)$ is the sup norm of the complex dilation of $f$. Now $\varphi$ is an analytic mapping and therefore a contraction in the hyperbolic metrics (Schwarz Lemma). The hyperbolic distance between 0 and $k$ in the unit disk is

$$
\rho_{\Delta}(0, k)=\log \left(\frac{k+1}{k-1}\right)=\log K .
$$

Hence

$$
\rho(z, f(z))=\rho(\varphi(0), \varphi(k)) \leq \log (K) .
$$

This proves the first part of Teichmüller's Theorem. To see the second part we need to construct an analytic disk $\varphi: \Delta \rightarrow \mathbb{C} \backslash\{0,1\}$ such that $\varphi(0)=z$ and $\varphi(k)=w$, where $k$ is as above. This gives a holomorphic motion of $\{0,1, \infty, z\}$ fixing 0,1 and $\infty$. The desired mapping $f$ is the extension of this motion at the point $k$. The analytic disk $\varphi$ is easily obtained by normalising the universal covering map $\Delta \rightarrow \mathbb{C} \backslash\{0,1\}$, since this map defines the hyperbolic metric of $\mathbb{C} \backslash\{0,1\}$.

We thank A. Hinkkanen for providing the following argument based on Teichmüller's original paper. We sketch the proof.

Theorem 4.1 implies Theorem 1.1. To conform to our notation, write $K=$ $(1+t) /(1-t)$, where $0 \leq t<1$. Let $r>0$ and $t \in[0,1)$ be fixed. Let $\rho_{\Delta}(z, w)$ denote the hyperbolic metric of the unit disk as above.

First note that if $-u=-u(t, r)<0$ is the unique negative real number such that $\rho_{\Delta}(-u,-r)=\log (1+t) /(1-t)=\log K$, then $\Psi_{\mathcal{Q} C}(t, r)=u>0$. Namely, by Theorem 4.1 we have

$$
\Psi_{\mathcal{Q} C}(t, r)=\max \{|z|: z \in \mathbb{C} \backslash\{0,1\}, \rho(z, w) \leq \log K \text { for some } w,|w|=r\} .
$$

The kind of asymmetry considerations that occur also in the proof of Teichmüller's theorem show that "more stretching" is possible starting at a point on the negative axis and going in the negative direction, and hence

$$
\max \{|z|: z \in \mathbb{C} \backslash\{0,1\}, \rho(z, w) \leq \log K \text { for some } w \text { with }|w|=r\}=u,
$$

where $u$ is as just defined. Thus it remains to be proved that $\Psi_{\mathcal{A}}(t, r)=u$.

If $\phi$ is an analytic function of $\Delta$ into $\mathbb{C} \backslash\{0,1\}$ then, as noted above, we have $\rho(\phi(z), \phi(w)) \leq \rho_{\Delta}(z, w)$ whenever $z, w \in \Delta$. Suppose that $|\phi(0)|=r$ and pick $z$ with $|z|=t$. Now $\rho(\phi(0), \phi(z)) \leq \rho_{\Delta}(0, z)=\rho_{\Delta}(0, t)=\log K$. By the above equation we have $|\phi(z)| \leq u$, and so $\Psi_{\mathcal{A}}(t, r) \leq u=\Psi_{\mathcal{Q} C}(t, r)$.

To prove that $\Psi_{\mathcal{A}}(t, r) \geq u$ (so that then $\Psi_{\mathcal{A}}(t, r)=u$ ), we only need to give an example of a function $\phi$ for which $\phi(0)=-r$ and $\phi(t)=-u$. We take $\phi$ to be a universal covering map of $\Delta$ onto $\mathbb{C} \backslash\{0,1\}$ with $\phi(0)=-r$ and with $\phi^{\prime}(0)$ being a negative real number. Then in fact, by symmetry and uniqueness considerations, $\phi$ takes real values on the real axis (that is, on the intersection of the real axis with $\Delta$ ). Since $\phi$ preserves hyperbolic distances locally and indeed when $z$ moves in $\Delta$ inside a fundamental region, that is, as long as the image curve does not wind around 0,1 , or or $\infty$ in $\mathbb{C} \backslash\{0,1\}$, it follows that $\rho(\phi(0), \phi(-t))=\rho_{\Delta}(0,-t)=\log K$ as soon as $\phi$ maps the segment $(0,1)$ one-to-one onto the negative real axis. That this is true is easily understood and is explained in texts on the modular function, 
e.g. Carathéodory, Theory of Functions, Vol. II [6]. This completes the proof of Theorem 1.1, assuming Theorem 4.1.

The referee points out that instead of stretching Teichmüller's argument one can also refer to Hempel's paper [11], where the maximal growth of the hyperbolic metric is studied. This together with Teichmüller's Theorem can also be used to prove Theorem 1.1.

\section{ANOTHER APPLiCATiON}

In this section we just want to present a simple application of the theory of holomorphic motions to a much studied problem in quasiconformal mappings. The statement of the result we shall prove is as follows.

Theorem 5.1. Let $\Omega$ be a planar domain with at least three boundary points and $\rho_{\Omega}$ the hyperbolic metric of $\Omega$. Suppose $z, w \in \Omega$ and

$$
\rho_{\Omega}(z, w) \leq \log K \text {. }
$$

Then there is a $K$-quasiconformal self-homeomorphism $f$ of $\Omega$ such that

- $f(\zeta)=\zeta$ for all $\zeta \in \partial \Omega$,

- $f(z)=w$.

Proof. Let $\eta: \Delta \rightarrow \Omega$ be a universal covering map with $\eta(0)=z$ and $\eta(t)=w$, where $\log (t+1) /(t-1)=\rho_{\Delta}(0, t)=\rho_{\Omega}(z, w)$. Consider the holomorphic motion of the set $\{z\} \cup \overline{\mathbb{C}} \backslash \Omega$ :

$$
h(\lambda, a)= \begin{cases}\eta(\lambda) & \text { if } a=z, \\ a & \text { if } a \notin \Omega .\end{cases}
$$

Since $\eta$ is valued in $\Omega$ and $h(0, a)=a$ for $a \in\{z\} \cup \overline{\mathbb{C}} \backslash \Omega$, we see that this holomorphic motion, and therefore its extension $H$ to $\overline{\mathbb{C}}$, preserves pointwise the boundary of $\Omega$. The quasiconformal mapping we seek is easily seen to be $H(t, \cdot)$ : $\overline{\mathbb{C}} \rightarrow \overline{\mathbb{C}}$

We do not know if the converse to this result holds, that is, if $\rho_{\Omega}(z, f(z)) \leq \log K$ for a quasiconformal mapping with boundary values given by the identity mapping.

\section{REFERENCES}

[1] L.V. Ahlfors, Lectures on Quasiconformal Mappings Van Nostrand 1966. MR 34:336

[2] S. Agard, Distortion theorems for quasiconformal mappings Ann. Acad. Sci. Ser. AI 41B (1968) 1-12. MR 36:5340

[3] G. Anderson and M.K. Vamanamurthy Distortion functions and quasiconformal mappings (to appear) N.Z.J. Math.

[4] K. Astala and G.J. Martin, Holomorphic Motions University of Helsinki, Preprint.

[5] A. Beurling and L.V. Ahlfors, The boundary correspondence under quasiconformal mappings, Acta Math. 96 (1956) 125-142. MR 19:258c

[6] C. Carathéodory, Theory of functions of a complex variable II, Chelsea, 1954. MR 16:346c

[7] A. Douady, Prolongement de Mouvements Holomorphes Séminaire Bourbaki 46 (775) (19934) 1-12. MR 95m:58104

[8] C. Earle, I. Kra and S. Krushkal, Holomorphic motions and Teichmüller spaces Trans. Amer. Math. Soc., 343, (1994), 927-948. MR 94h:32035

[9] F. W. Gehring and G.J. Martin, Schottky's theorem, holomorphic motions and discrete groups, Computational Methods and Function Theory (to appear)

[10] J. A. Hempel, Precise bounds in the theorems of Schottky and Picard, J. London Math. Soc. 21 (1980) 279-286. MR 81i:30053 
[11] J. A. Hempel, The Poincaré metric of the twice punctured plane and the theorems of Landau and Schottky, J. London Math. Soc. 2 (1979) 435-445. MR 81c:30025

[12] O. Lehto, Univalent functions and Teichmüller spaces, Springer-Verlag 1987. MR 88f:30073

[13] O. Lehto and K. I. Virtanen, Plane quasiconformal mappings, Springer-Verlag 1973. MR 49:9202

[14] O. Lehto, K. I. Virtanen and J. Väisälä, Contribution to the distortion theory of quasiconformal mappings Ann. Acad. Sci. Fenn. Ser. AI 273 (1959) 1-14. MR 23:A321

[15] R. Mañé, P. Sad and D. Sullivan, On the dynamics of rational maps, Ann. Sci. École Norm. Sup. 16 (1983) 193-217. MR 85j:58089

[16] Z. Slodkowski, Holomorphic motions and polynomial hulls, Proc. Amer. Math. Soc. 111 (1991) 347-355. MR 91f:58078

[17] O. Teichmüller, Untersuchungen über konforme und quasiconforme Abbildung Deutsche Math 3 (1938) 621-678 (or see Collected works, Ed. L.V. Ahlfors and F.W. Gehring, Springer Verlag $(1982))$

Department of Mathematics, Australian National University, Canberra, ACT, AusTRALIA

Department of Mathematics, University of Auckland, Auckland, New Zealand

E-mail address: martin@math.auckland.ac.nz 